\title{
ANÁLISE INELÁSTICA DE SEGUNDA ORDEM EM PÓRTICOS PLANOS DE AÇO
}

\author{
Second order inelastic analysis of steel frames
}

\author{
Alex Sander Clemente de Souza', Margot Fabiana Pereira²; Silvana De Nardin ${ }^{3}$
}

Recebido em 26 de janeiro de 2016; recebido para revisão em 07 de julho de 2016; aceito em 08 de agosto de 2016; disponivel on-line em 09 de agosto de 2016.

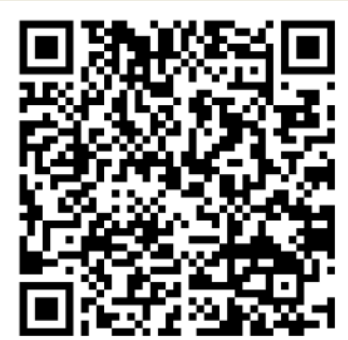

\section{PALAVRAS CHAVE:}

Análise estrutura; Análise de segunda ordem;

Não-linearidade do material;

Não-linearidade geométrica;

Efeito P-Delta.

\section{KEYWORDS:}

Structural analysis; Second order analysis; Nonlinearity of material; Geometric nonlinearity; P-Delta Effect.

* Contato com os autores:

1e-mail: alex@ufscar.br

Doutor em Eng. de Estruturas, Prof. Associado do Dep. De Eng. Civil da Universidade Federal de São Carlos - UFSCar.

2e-mail: margot.pereira@usp.br

Doutoranda no Programa de Pós-Graduação em Engenharia de Estruturas da Escola de Engenharia de São Carlos - EESC-USP.

3 e-mail: snardin@ufscar.br

ISSN: 2179-0612 D.O.I. 10.5216/reec.V12i3.39540
RESUMO: Na análise estrutural de edifícios é necessária e exigida em códigos de projeto a consideração do comportamento não-linear do material e da estrutura para determinação de esforços e deslocamentos. O desenvolvimento de novos softwares permite a realização de análises mais rigorosas, como a análise avançada, em que é possível avaliar a capacidade resistente e a estabilidade da estrutura dispensando a verificação dos elementos isolados e ainda considerando os efeitos não-lineares, sejam eles físicos e/ou geométricos. Por outro lado, os códigos normativos ainda permitem procedimentos simplificados para a análise estrutural, embora seja relativamente comum o uso de métodos mais avançados de análise, como por exemplo, o Método dos Elementos Finitos. Pretende-se, com este estudo, fornecer subsídios para a compreensão dos códigos normativos e a importância da escolha do modelo de análise estrutural na fase de projeto. Neste contexto, a fim de exemplificar, os efeitos das não-linearidades geométrica e física sobre o comportamento da estrutura foram avaliados pórticos planos em aço por meio de modelagem avançada. Além disso, uma modelagem com elementos finitos de barra / mola foi desenvolvida e os resultados obtidos se mostraram compatíveis com aqueles dos modelos tridimensionais onde os perfis foram modelados com elementos de chapa. Desta forma contribui-se para ampliar o estado da arte sobre análise estrutural de forma integrada com o dimensionamento de estruturas metálicas.

ABSTRACT: In structural analysis of buildings it is necessary and required in design codes include non -linear effects on the material and structure for determining forces and displacements. The development of new software allows more rigorous analyzes, such as the advanced analysis; it is possible to assess the strength and stability of a structure excusing the verification of separate structural elements and considering the nonlinear effects. The design codes allow structural analysis by simplified procedures. However, it has been used advanced analysis methods such as the Finite Element Method. The aim of this study provide support for the normative codes and the importance of choosing the structural analysis model in the design phase. In order to illustrate the effects of geometry and physical nonlinearities on the behavior of the structure was evaluated plane frames steel through advanced modeling. In addition, a finite element modeling bar / spring was developed and the results obtained were consistent with those of three-dimensional shell models. In this way it contributes to extend the state of the art structural analysis integrated with the design of steel structures.

\section{(A. S. C. de Souza)}

(M. F. Pereira)

(S. D. Nardin)

a. do Dep. De Eng. Civil da Universidade Federal de São Carlos - UFSCar. 


\section{INTRODUÇÃO}

A avaliação do comportamento estrutural, determinação dos esforços, deslocamentos e análise da estabilidade devem ser feitas por meio da análise estrutural mais adequada para cada caso. A análise estrutural depende das características de rigidez e do comportamento das seções, das imperfeições de fabricação e montagem, do comportamento das ligações e, principalmente, da estabilidade dos elementos e da estrutura como um todo (SOUZA e PERREIRA, 2009). Assim, a escolha do modelo de análise mais adequado a uma dada estrutura deve considerar todos esses aspectos. Do ponto de vista da prática de projeto, ainda não é possível incluir todos esses efeitos nos modelos de análise estrutural, pois algumas técnicas ainda necessitam de amadurecimento e, em geral, exigem maior tempo de processamento e análise dos resultados e, finalmente, uma parcela significativa dos engenheiros não está familiarizada com essas técnicas (DORIA ,2007).

Além disso, a análise estrutural é uma etapa importante do projeto estrutural impactando o dimensionamento final e, consequentemente, o desempenho e os custos. Desta forma pesquisas sobre os procedimentos, técnicas e ferramentas de análise estrutural são necessárias e plenamente justificáveis.

\subsection{OBJETIVOS}

Neste contexto, este artigo tem como principal objetivo tecer uma discussão sobre os principais aspectos da modelagem estrutural aplicada a pórticos planos de aço, bem como a análise crítica da resposta estrutural obtida a partir das diferentes formas de modelagem. Para esse fim, exemplos de pórticos planos em aço foram modelados segundo as recomendações da norma brasileira NBR 8800 (ABNT, 2008) e, também com o uso de modelos de análise mais rigorosos incluindo a rigidez das ligações e as não-linearidades física e geométrica. Foi desenvolvida uma modelagem em elementos finitos de casca e outra mais simples com elementos de barra. Ambas foram realizadas utilizando o pacote computacional Ansys 15 e os detalhes dos pórticos e das variáveis analisadas são detalhados mais adiante.

\section{CONCEITUAÇÃO TEÓRICA}

Atualmente, várias pesquisas como, por exemplo, Doria (2007), Silva (2004), Souza e Pereira (2009) e Pereira e Souza (2009) buscam incluir na análise estrutural condições reais da estrutura, de modo que estas reproduzam de maneira mais coerente os resultados obtidos em ensaios com modelos físicos. Entre estes aspectos, estão as não linearidades geométrica e física. A análise com nãolinearidade geométrica (análise em segunda ordem) considera o equilíbrio da estrutura na posição deformada o que influencia na magnitude dos esforços gerando, inclusive, esforços adicionais que alteram os próprios deslocamentos, caracterizando uma relação não linear entre esforços e deslocamentos. Esses efeitos adicionais também são denominados, na linguagem de projeto, de efeitos de 2a ordem ou efeitos P-“delta". Os efeitos P-"delta" podem estar associados aos deslocamentos da estrutura ou às deformações dos elementos; no primeiro caso são denominados de efeitos de 2 a ordem globais (efeitos P- $\Delta$ - Figura 1a), e, no segundo, efeitos de 2 a ordem locais (efeitos $P$ $\delta$ - Figura 1b). De modo geral, ambos os efeitos citados são decorrentes de mudanças na geometria da estrutura, ou seja, da não linearidade geométrica.

Já a não-linearidade física está relacionada com o comportamento tensão $\mathrm{x}$ deformação do material que compõe o elemento estrutural e pode ser representada por um modelo constitutivo que relacione adequadamente tensão e deformação e este modelo pode ser introduzido na análise estrutural por meio de análise incrementaliterativa. 0 método incremental-iterativa também é utilizado na análise não-linear geométrica comentada a seguir.

\subsection{A NÃO LINEARIDADE GEOMÉTRICA}

$\mathrm{Na}$ literatura científica encontram-se vários métodos para incluir os efeitos da não linearidade geométrica na análise estrutural. Reis e Camotim (2001) classificam estas metodologias em: análises rigorosas, aproximadas e modificadas. 


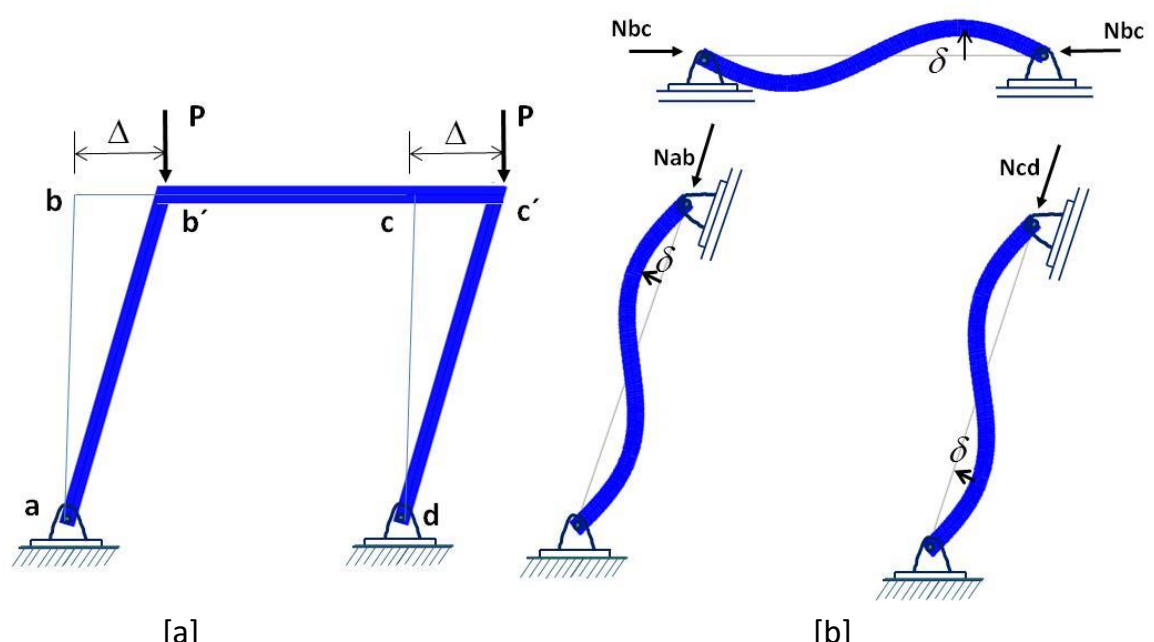

FIGURA 1: Efeitos de segunda ordem. [a] Efeitos P- $\Delta$ [b] Efeitos P- $\delta$. FONTE: REIS E CAMOTIM (2001).

Nas análises rigorosas são levadas em conta as posições instantâneas para cada estágio de carregamento. Muitos pacotes computacionais foram desenvolvidos para realização deste tipo de análise e a maioria deles utiliza o Método dos Elementos Finitos como ferramenta (GALVÃO, 2000). Há também pacotes comerciais como o ANSYS e SAP 2000 que permitem a realização de análises rigorosas.

Já nas análises aproximadas os efeitos da não-linearidade geométrica são inseridos indiretamente por meio da redução da rigidez ou aplicação de forças nocionais como, por exemplo, o Método das Forças Laterais Equivalentes. Este processo consiste em substituir um problema não-linear por vários problemas lineares sucessivos. As forças nocionais devem ser somadas aos carregamentos laterais, como o carregamento de vento, e então a estrutura é novamente analisada em 1a ordem e os novos deslocamentos são gerados. $O$ processo deve se repetir até a convergência dos deslocamentos. É importante observar que este método, popularmente conhecido como processo $\mathrm{P}-\Delta$, leva em conta apenas os efeitos da não linearidade geométrica global (WOOD et al. 1976).

Finalmente, nas análises modificadas são utilizados coeficientes de amplificação dos efeitos de $1 \underline{a}$ ordem. Um destes métodos, denominando Método de amplificação dos momentos fletores utiliza os coeficientes $B_{1}$ e $B_{2}$ como amplificadores. Trata-se de um método bastante conhecido e que considera os efeitos da não linearidade geométrica globais e locais separadamente; parâmetro $B_{1}$ para efeitos locais e $B_{2}$ para os efeitos globais. As normas NBR 8800 (ABNT, 2008) e AICS LRFD (2005) permitem a utilização desse procedimento simplificado de análise.

Vários pesquisadores vêm estudando os efeitos da não linearidade geométrica em estruturas de aço e estes estudos vão desde aspectos puramente teóricos, desenvolvimento de ferramentas numéricas a aplicações em projetos visando aprimorar os procedimentos normativos. Neste sentido, vale destacar os estudos de DORIA (2007), SILVA (2004), SOUZA e PEREIRA (2009) e PEREIRA E SOUZA (2009).

\subsection{A NÃO LINEARIDADE FÍSICA}

A não linearidade física pode ser incluída na análise estrutural por meio de diversos métodos, entre eles o método das rótulas plásticas e o método da zona plástica, ambos estudados por CAMILO (1991), KING (1994), ALVARENGA (2005) e MACHADO (2005), entre outros. A análise por rótulas plásticas é uma análise inelástica em primeira ordem em que as deformações são consideradas concentradas em uma região do elemento estrutural. Isto é, a partir do instante em que as fibras de uma seção atingem a resistência ao escoamento esta seção passa a se comportar como uma rótula pontual para qualquer acréscimo no carregamento. $\mathrm{O}$ aparecimento sucessivo de rótulas plásticas pode levar a estrutura ao colapso por formação de mecanismo. 
Outro método de análise inelástica é o método da zona plástica no qual há a substituição da rótula pontual por uma região tridimensional onde atuam tensões superiores à resistência ao escoamento do aço. Assim, considera-se a plasticidade distribuída ao longo do comprimento e da área da seção transversal de um elemento. No contexto do método dos elementos finitos, a seção é dividida em vários subelementos (faixas) nos quais são avaliadas as tensões e as deformações. Neste método também é possível incluir tensões residuais, imperfeições geométricas e rigidez das ligações. Por todas estas possibilidades, o método da zona plástica é um método de análise avançada segundo as definições de ALVARENGA e SILVEIRA (2006), CHEN (2008), THAI e KIM (2009) e LIU, LIU e CHAN (2012).

Outra forma de considerar a não linearidade física é a realização de uma análise não linear incremental-iterativa introduzindo o modelo constitutivo representativo do material e adotando um critério de ruptura adequado para este material. O critério de ruptura, ou superfície de interação, ou superfície de plastificação, também pode ser utilizado no método de rótulas plásticas, como no caso do algoritmo de retorno radial, onde também é utilizado o método incremental iterativo.

Acredita-se que a tendência na análise estrutural é a adoção da chamada análise avançada. Segundo Gomes (2005), entende-se por análise avançada todo método capaz de verificar, de maneira conjunta, a resistência e a estabilidade de uma estrutura de modo que seja eliminada a necessidade de dimensionamento dos seus elementos isoladamente. Obviamente, neste método devem estar incluídas todas as não linearidades e o comportamento das ligações. Algumas normas como o AISC-LRFD:2005 já preconizam o projeto estrutural utilizando o método avançado de análise.

\subsection{CRITÉRIO NORMATIVO - NBR 8800 (ABNT, 2008)}

Os códigos normativos, em geral, tratam a análise estrutural de forma diversa no que se refere à classificação quanto a deslocabilidade. Essa classificação pode ser feita por meio da relação entre os deslocamentos laterais de cada andar, em relação à base, obtidos em análise não-linear geométrica (análise de $2 \circ$ ordem) e os obtidos em análise linear (análise de $1 \underline{a}$ ordem) para as combinações últimas de projeto. No caso da NBR 8800 - (ABNT, 2008) esta relação pode ser aproximada pelo parâmetro $B_{2}$ apresentado na Equação (1).

$$
B_{2}=\frac{1}{1-\frac{1}{R s} \cdot \frac{\Delta_{h}}{h} \cdot \frac{\sum N_{S d}}{\sum H_{S d}}}
$$

Sendo:

$R_{S}=0,85$ para estruturas constituídas apenas por pórticos onde a estabilidade é garantida pela rigidez à flexão das barras e pela capacidade de transmitir momento das ligações e $R_{S}=1,0$ para as demais estruturas;

$\Delta \mathrm{h}$ : deslocamento inter-pavimento em cada andar, obtido em análise de 1aㅡordem;

$\sum H_{S d}$ : força cortante no andar, produzida pelas forças horizontais de cálculo;

$\sum N_{S d}$ : carga gravitacional total no andar considerado;

h: altura do andar.

Uma vez calculado o parâmetro $B_{2}$, definem-se quais efeitos de não linearidade devem ser considerados no modelo de análise, como a não-linearidade física, não-linearidade geométrica e imperfeições iniciais. No Quadro 1 apresenta-se, resumidamente, os procedimentos de análise recomendados pela NBR 8800 (ABNT, 2008) em função da classificação quanto a deslocabilidade.

Os procedimentos para análise não linear geométrica segundo as normas NBR 8800 - (ABNT, 2008), AISC-LRFD:2005 e EC 3:1994 têm origens comuns, embora aplicadas com diferentes metodologias. Estas normas apresentam procedimentos simplificados para análise não linear geométrica, embora permitam o uso de análises mais refinadas. 
QUADRO 1: Classificação da estrutura quanto a deslocabilidade.

\begin{tabular}{|c|c|c|c|}
\hline CLASSIFICAÇÃO & $\begin{array}{l}\text { Pequena deslocabilidade } \\
\qquad B_{2} \leq 1,1 \\
\text { Em todos os pavimentos. }\end{array}$ & $\begin{array}{l}\text { Média deslocabilidade } \\
\qquad 1,1<B_{2} \leq 1,4\end{array}$ & $\begin{array}{l}\text { Grande deslocabilidade } \\
\qquad B_{2}>1,4\end{array}$ \\
\hline $\begin{array}{l}\text { Modelos de } \\
\text { Análise }\end{array}$ & $\begin{array}{c}\text { Análise em 1a ordem se as } \\
\text { forças axiais de cálculo nas } \\
\text { barras que participam do } \\
\text { sistema de estabilidade lateral } \\
\text { não superam } 50 \% \text { da força axial } \\
\text { de plastificação. } \\
\text { Imperfeições geométricas } \\
\text { iniciais devem ser incluídas na } \\
\text { análise. }\end{array}$ & $\begin{array}{c}\text { Análise de } 2 \text { a ordem } \\
\text { simplificada utilizando os } \\
\text { parâmetros } B_{1} \text { e } B_{2} \\
\text { calculados com a rigidez } \\
\text { reduzida dos elementos da } \\
\text { estrutura. } \\
\text { As forças nocionais não } \\
\text { devem ser somadas às ações } \\
\text { laterais. }\end{array}$ & $\begin{array}{l}\text { Deve ser feita análise de } 2^{\text {a }} \\
\text { ordem rigorosa incluindo } \\
\text { imperfeições geométricas e } \\
\text { de material. }\end{array}$ \\
\hline $\begin{array}{l}\text { Imperfeições } \\
\text { do Material }\end{array}$ & NÃO CONSIDERAR & \multicolumn{2}{|c|}{$\begin{array}{l}\text { Reduzir a rigidez à flexão e à compressão dos elementos } \\
\text { para } 0,8 \mathrm{El} \text { e } 0,8 \mathrm{EA} \text {, respectivamente. }\end{array}$} \\
\hline
\end{tabular}

FONTE: Autoria própria.

Os processos de análise recomendados pelas normas ABNT NBR 6118:2014 e EC 3:1994 utilizam procedimentos mais simples. Já a NBR 8800 (ABNT, 2008) e o AISC-LRFD:2005, ambas normas voltadas para o dimensionamento de estruturas de aço, apresentam procedimentos mais claros do ponto de vista conceitual uma vez que separam os efeitos locais e globais, bem como os efeitos das imperfeições geométricas (forças horizontais fictícias) e de material (redução da rigidez).

\section{METODOLOGIA}

No presente estudo foram desenvolvidos diferentes tipos de análise estrutural utilizando três modelos de pórticos planos, simulados com o pacote computacional ANSYS 15 via Método dos Elementos Finitos. Os três exemplos estudados foram denominados Pórtico de Vogel, Pórtico 1 e

Pórtico 2. Nas Figuras 2, 3 e 4 apresentam-se as principais características dessas estruturas como geometria, seções transversais dos elementos e carregamento de cálculo.

Os resultados obtidos nas análises do Pórtico de Vogel, que é um pórtico plano estudado por diversos autores, foram utilizados para calibração da metodologia proposta neste estudo.
Os carregamentos indicados nos demais pórticos representam carregamentos usuais de projeto.

As estruturas foram analisadas segundo quatro modelos de análise:

- Modelo A: Análise elástica em 1ạ ordem;

- Modelo B: Análise elástica em 2aㅡ ordem;

- Modelo C: Análise inelástica em 2a ordem;

- Modelo D: Análise inelástica em 2a ordem incluindo a rigidez das ligações. Além disso, as estruturas também foram analisadas segundo os procedimentos simplificados apresentados na NBR 8800 (ABNT, 2008) com base nos coeficientes de modificação $B_{1}$ e $B_{2}$; este modelo de análise é denominado Método de Amplificação de Esforços (Anexo D da NBR 8800 (ABNT, 2008)). Para o Modelo $D$, no qual é incluída a rigidez das ligações, foram adotados os valores de rigidez inicial $\left(S_{i}\right)$ obtidos experimentalmente por Ribeiro (1997) para ligações semelhantes às adotadas aqui. A partir das Equações 2 e 3, desenvolvidas por Santos (1998), é possível estimar a rigidez inicial de ligações com chapa de topo com uma boa correlação com os resultados experimentais de RIBEIRO (1997). 

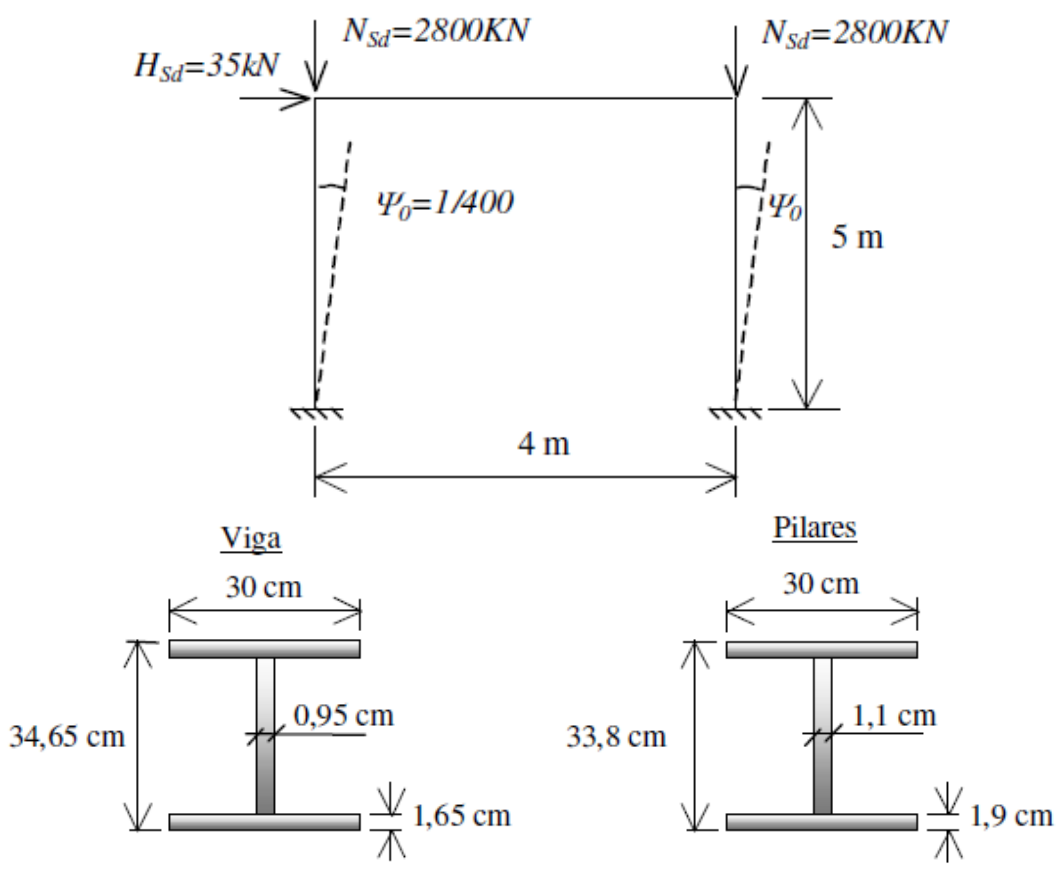

FIGURA 2: Pórtico de Vogel.

FONTE: Autoria própria.

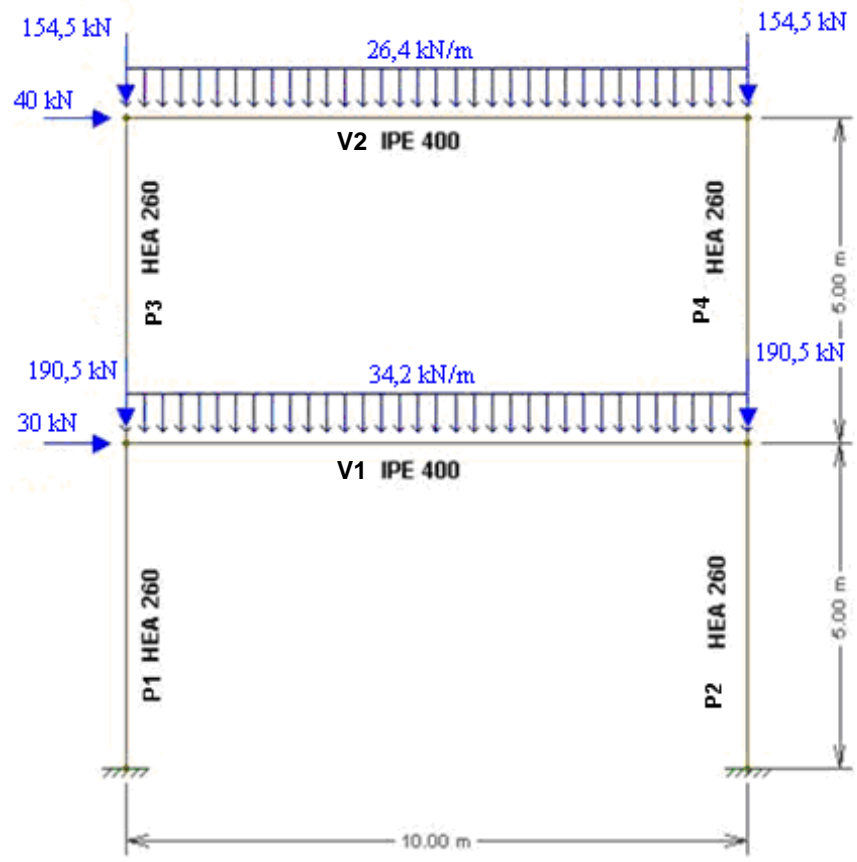

FIGURA 3. Pórtico 1.

FONTE: Autoria própria. 


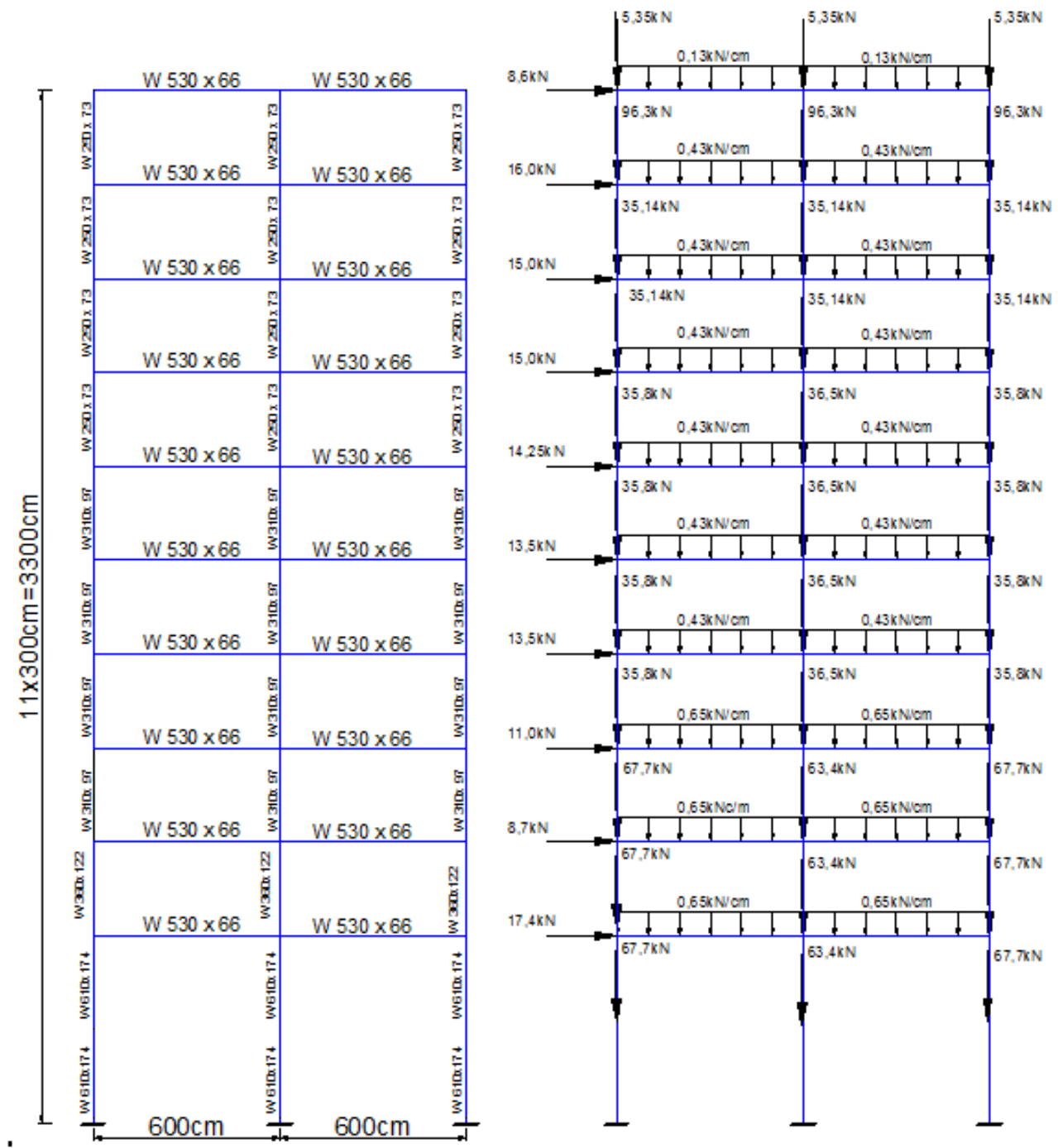

FIGURA 4: Pórtico 2.

FONTE: Autoria própria.

$$
\begin{aligned}
S_{i} & =\frac{24 E I K\left(H-t_{f}\right)^{2}}{12 E I+K b^{3}} \\
K & =2 \cdot \frac{E \cdot A_{p}}{L_{p}} \cdot \frac{t_{c h}}{b_{c h}}
\end{aligned}
$$

$t_{f}$ : espessura do perfil da mesa da viga $(\mathrm{cm})$;

$t_{c h}$ : espessura da chapa de topo $(\mathrm{cm})$;

$A_{p}$ : área do parafuso $\left(\mathrm{cm}^{2}\right)$;

$\mathrm{L}_{\mathrm{p}}$ : comprimento do parafuso $(\mathrm{cm})$;

E: módulo de elasticidade do material $\left(\mathrm{kN} / \mathrm{cm}^{2}\right)$;

Eq. [3]

I: Momento de inércia da seção $\left(\mathrm{cm}^{4}\right)$.

Na Tabela 1 apresentam-se os valores de

Sendo:

$\mathrm{H}$ : altura do perfil da viga nas ligações $(\mathrm{cm})$;

$\mathrm{b}_{\mathrm{ch}}$ : largura da chapa de ligações $(\mathrm{cm})$; rigidez inicial adotados para cada um dos modelos analisados no presente estudo.

\begin{tabular}{ccc}
\hline & TABELA 1: Valores de rigidez inicial da ligação. \\
& $\begin{array}{c}\text { Rigidez ligação } 01 \\
\text { (kN.m/rad) }\end{array}$ & $\begin{array}{c}\text { Rigidez ligação } 02 \\
\text { (kN.m/rad) }\end{array}$ \\
\hline Pórtico 1 & 50666,7 & 42222,2 \\
\hline Pórtico 2 & 73228,0 & 125504,0 \\
\hline
\end{tabular}


O Pórtico de Vogel foi analisado no pacote computacional ANSYS. Na primeira modelagem os elementos vigas e pilares foram discretizados com elementos de casca Shell 181, com uma malha retangular uniforme com 3600 elementos finitos. Assim foi possível modelar e melhor representar a região da ligação viga-pilar. Posteriormente, Pórtico de Vogel foi modelado com elementos de barra Beam 24, sendo cada barra dividida em 5 elementos finitos. Neste modelo foi utilizado o elemento Combin 40 para simular a rigidez da ligação vigapilar. Com os resultados desses dois modelos foi possível calibar o comportamento das ligações e para os demais exemplos foram simulados utilizando somente os elementos de barra Beam 24 para discretização de vigas e pilares (sendo cada barra dividida em 5 elementos) e o Combin 40 para as ligações.

Para análise não linear física foi adotado um diagrama tensão $\mathrm{x}$ deformação Multi-linear (Isotropic Hardening Plasticity MISO - ANSYS) Figura 5.
Foram adotados módulo de elasticidade longitudinal $E=20500 \mathrm{kN} / \mathrm{cm}^{2}$ e resistência ao escoamento $\mathrm{f}_{\mathrm{y}}=25 \mathrm{kN} / \mathrm{cm}^{2}$ para as propriedades do aço.

A seguir, os principais resultados obtidos são apresentados e discutidos.

\section{ANÁLISE DOS RESULTADOS}

\subsection{PÓRTICO DE VOGEL}

O Pórtico de Vogel foi utilizado para calibrar a metodologia utilizada neste estudo. Inicialmente foi desenvolvida uma modelagem com elementos de casca (Shell 181) considerando as não linearidades geométrica e física e melhor representando a região da ligação viga-pilar. Os resultados foram comparados aos obtidos por Kim e Lee (2001) e Gomes (2005) para a mesma estrutura, obtendo-se correlações satisfatórias (Figura 6) para os deslocamentos horizontais no topo do pórtico.

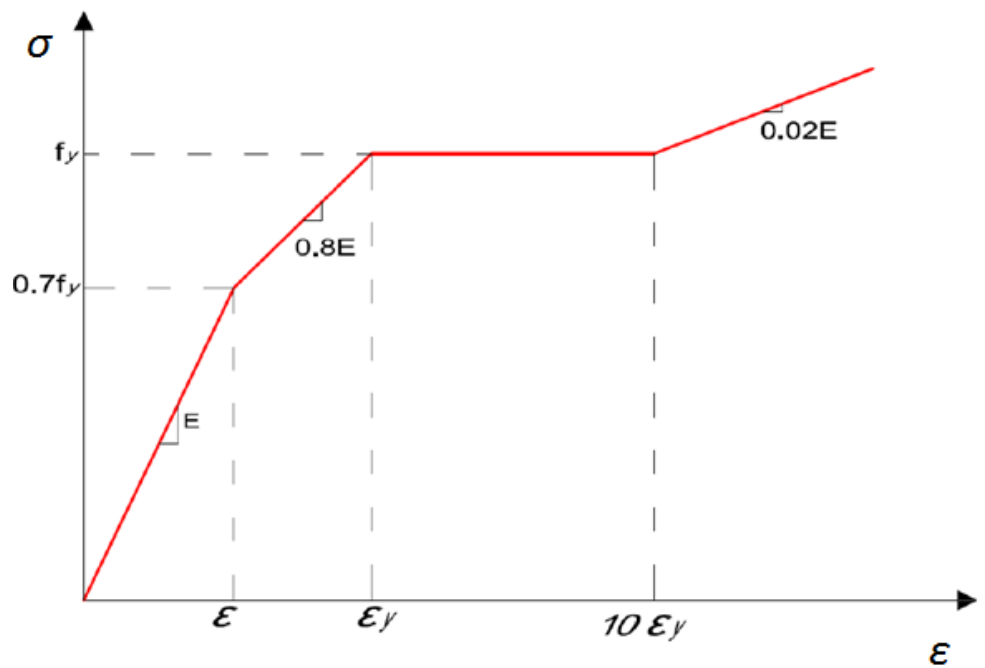

FIGURA 5: Modelo Constitutivo - Gráfico Tensão $(\sigma)$ x Deformação $(\varepsilon)$. FONTE: Autoria própria. 


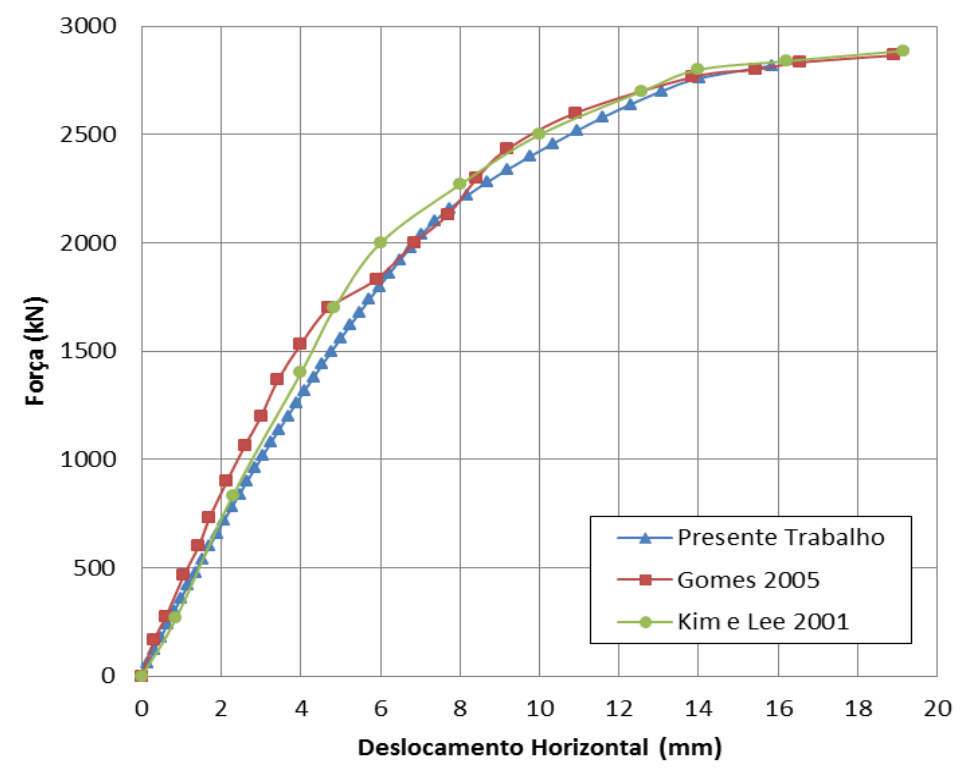

FIGURA 6: Comparação das curvas Força-Deslocamento do pórtico de Vogel utilizando elemento de casca. FONTE: Autoria própria.

A utilização de elementos de casca torna a modelagem trabalhosa, sendo necessário grande esforço computacional o que se torna inviável para o dia-a-dia de projeto. Desta forma, buscou-se desenvolver modelos com elementos de barra que permitissem obter resultados compatíveis com os modelos de casca porém com menor tempo de modelagem e processamento. Assim, foi desenvolvida uma nova modelagem com o elemento finito de barra Beam 24, para a discretização de pilares e vigas e Combin 40 para simular as ligações e sua rigidez. $\mathrm{O}$ valor da rigidez das ligações foi definido em função dos resultados obtidos na modelagem com elementos de casca, ou seja, foram utilizados diferentes valores de rigidez inicial da ligação a fim de obter resultados de deslocamentos próximos aos obtidos com o modelo de casca.

No gráfico da Figura 7 apresenta-se o comportamento Força aplicada versus Deslocamento horizontal no topo do pórtico em função da rigidez inicial da ligação. Observa-se boa correlação entre os resultados obtidos via modelo com elementos de casca e com elementos de barra, especialmente para a rigidez inicial de $2000 \mathrm{kNm} / \mathrm{rad}$. Observa-se também diferenças significativas no modelo com nós rígidos em relação aos demais o que torna clara a importância da rigidez da ligação para representar o comportamento e a resposta estrutural.

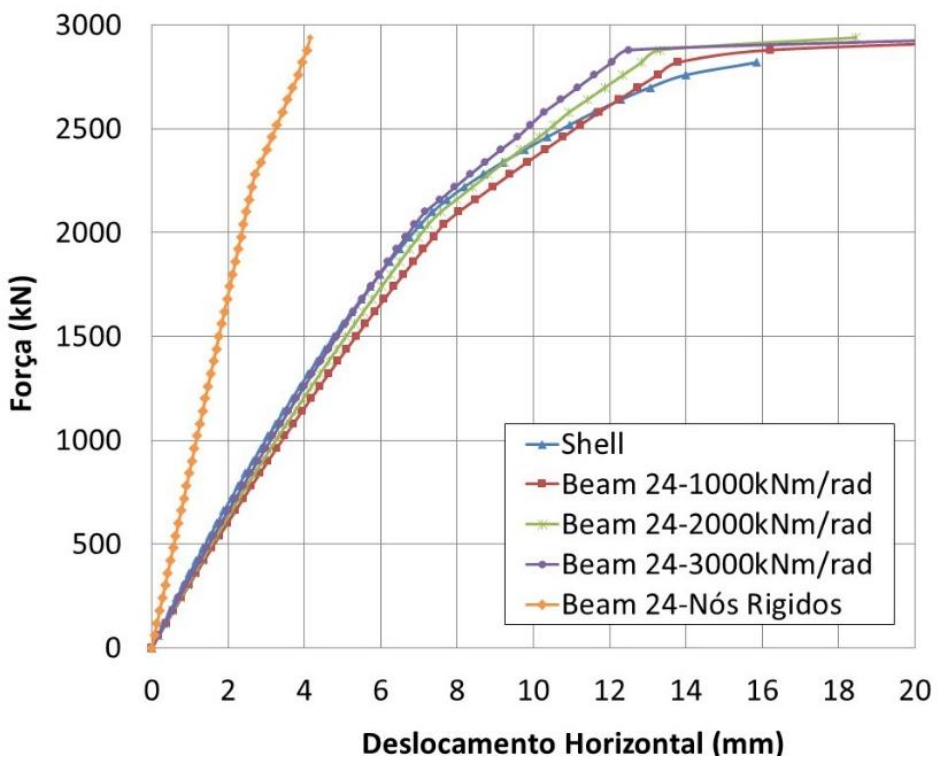

FIGURA 7: Comparação das curvas Força-Deslocamento em função da rigidez das ligações. FONTE: Autoria própria. 
A partir deste exemplo, verificou-se que é possível desenvolver modelos com elementos de barra que simulem de maneira satisfatória o comportamento da estrutura com menor esforço computacional, desde que sejam consideradas na análise: a rigidez das ligações, a não linearidade física e a não linearidade geométrica. Com base nestes resultados, as demais estruturas foram analisadas apenas com elementos de barra.

\subsection{PÓRTICO 1}

Para o Exemplo 1 foram desenvolvidos modelos numéricos utilizando os elementos de barra Beam24, já utilizados para o Pórtico de Vogel. Foram feitas considerações para as ligações vigapilar rígidas e semirrígidas sendo que para estas últimas foi utilizado o elemento Combin 40. O gráfico na Figura 8 apresenta os resultados de Força aplicada versus Deslocamento horizontal no topo do pórtico em função dos modelos de análise adotados. Vale lembrar que para o Modelo $D$ foram utilizados os dois valores de rigidez $K_{1}$ e $K_{2}$ apresentados na Tabela 1. Para os dois valores de rigidez da ligação a estrutura foi classificada como de pequena deslocabilidade segundo os critérios da NBR 8800 (ABNT, 2008).

Pode-se observar que os deslocamentos obtidos em análise elástica são inferiores aos obtidos nas demais análises. Além disso, verifica-se que os deslocamentos são maiores à medida que o modelo de análise torna-se mais rigoroso, ou seja, quando se incorporam as não linearidades, imperfeições e rigidez da ligação.

Outra comparação pertinente se refere aos esforços solicitantes. Os momentos fletores obtidos para cada um dos modelos de análise foram comparados entre si e comparados com os valores obtidos pelos procedimentos da NBR 8800 (ABNT, 2008). Na Figura 9 apresenta-se a comparação dos momentos fletores nos trechos inicial e final dos pilares do Pórtico 1 obtidos via ANSYS com os obtidos pelo procedimento normativo da NBR 8800 (ABNT, 2008). No gráfico da Figura 9 os elementos 1 e 2 são os pilares do 1 o pavimento e os elementos 23 e 24 os pilares do 2o pavimento do Pórtico 1.

Observa-se que os momentos fletores obtidos pelos procedimentos da NBR 8800 (ABNT, 2008) são, na maior parte das vezes, superiores aos obtidos pelos modelos de análise propostos. Os momentos calculados segundo os procedimentos da norma se aproximam mais dos obtidos para os Modelos A e B, que são análise elástica linear e não linear geométrica, respectivamente. Há uma divergência maior entre os resultados normativos e os obtidos nos Modelos $C$ e $D$, pois estes consideram a não linearidade física e/ou semirrigidez das ligações o que é considerado muito simplificadamente no procedimento normativo.

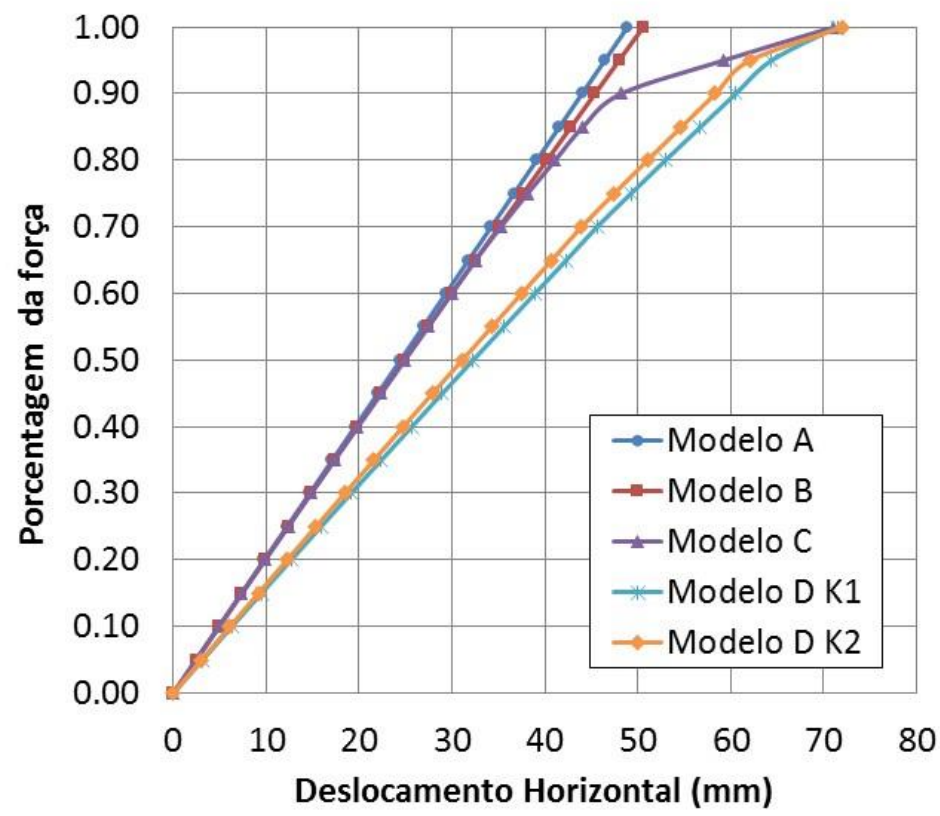

FIGURA 8: Força aplicada versus deslocamento para o Pórtico 1. FONTE: Autoria própria. 


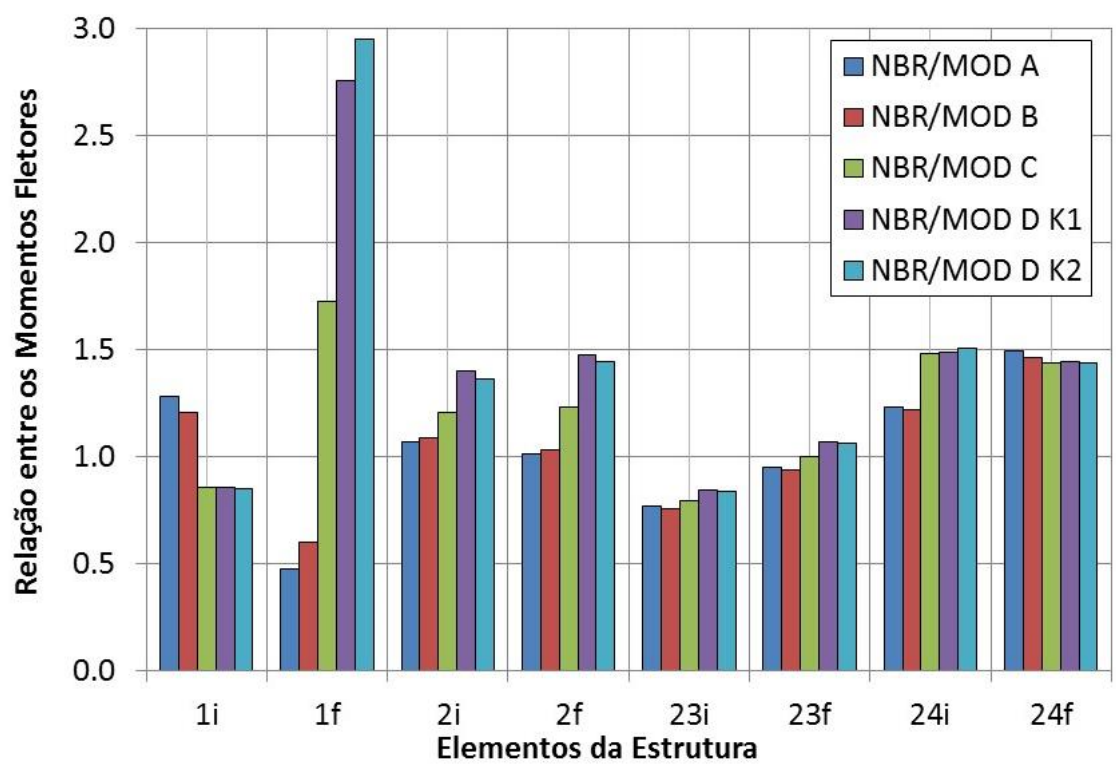

FIGURA 9. Comparação entre os Momentos obtidos via ANSYS e pela NBR 8800 (ABNT, 2008). FONTE: Autoria própria.

\subsection{PÓRTICO EXEMPLO 2}

O Pórtico 2 trata-se de um pórtico de 2 tramos e 11 pavimentos, sendo o primeiro pavimento com pé-direito duplo. Foram utilizadas as mesmas considerações de análise do exemplo anterior inclusive os mesmos valores de rigidez inicial das ligações apresentados na Tabela 2. No gráfico da Figura 10 apresenta-se o comportamento
Força aplicada versus Deslocamento horizontal no topo do pórtico e mostra comportamento praticamente linear para estas curvas. Além disso, verificou-se que a consideração da rigidez das ligações resultou em deslocamentos significativamente maiores e não modificou a classificação da estrutura quanto a deslocabilidade segundo os critérios da NBR 8800 (ABNT, 2008).

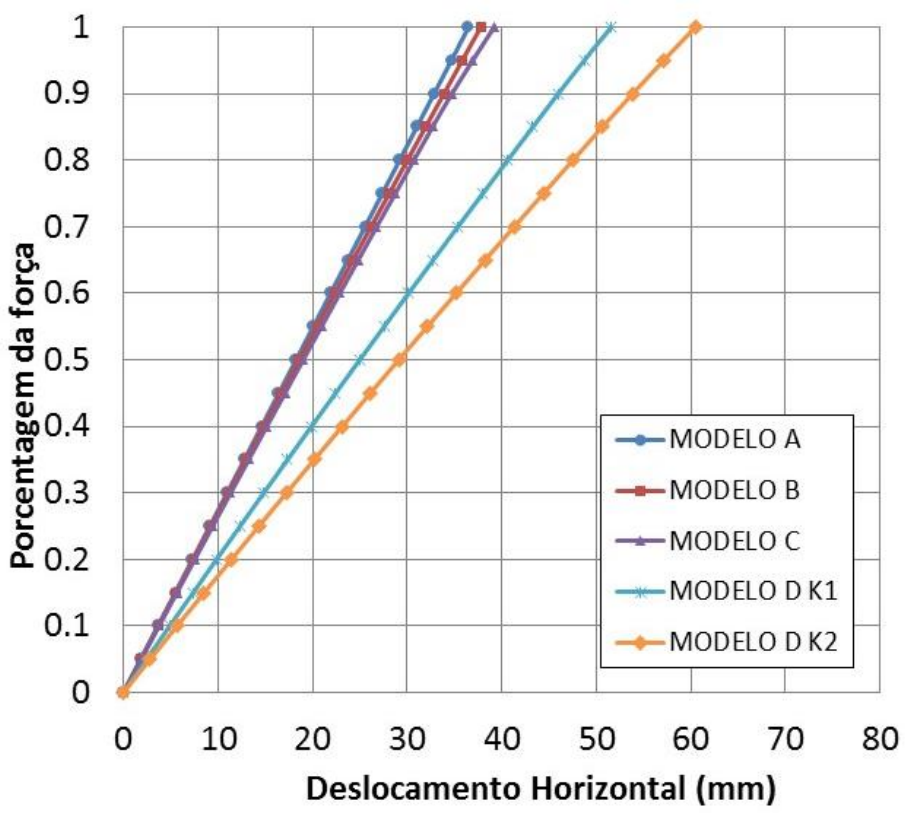

FIGURA 10: Deslocamentos x Incremento de Força para o Pórtico 2. FONTE: Autoria própria. 
Os esforços de segunda ordem foram obtidos nos modelos de análise $A, B, C$ e D e também por meio do procedimento simplificado da NBR 8800 (ABNT, 2008). A estrutura foi classificada como de média deslocabilidade já que o máximo valor do parâmetro $B_{2}$ foi de 1,13. Desta forma, foram consideradas também as imperfeições do material de forma simplificada como recomenda a NBR 8800 (ABNT, 2008).

No gráfico da Figura 11 apresenta-se a comparação entre os momentos fletores para os modelos de análise numérica e os valores obtidos pelos procedimentos da ABNT NBR 8800:2008. Observa-se boa correlação entre os resultados, de modo que a relação entre os momentos obtidos pelo procedimento normativo e os da modelagem numérica ficou próxima de 1,0 para a maior parte dos elementos da estrutura. As maiores divergências entre os resultados obtidos pela NBR 8800 (ABNT, 2008) e pelos Modelos D-K $1_{1}$ e $D-K_{2}$. Os Modelos D-K $K_{1}$ e $D-K_{2}$ consideram não linearidades física e geométrica além da rigidez das ligações e estes fatores explicam as diferenças em relação aos procedimentos simplificados da NBR 8800 (ABNT, 2008). No gráfico da Figura 11 as barras de 1 a 11 correspondem aos pilares da esquerda, barras de 12 a 22 os pilares centrais e barras de 23 a 33 aos pilares da direita.

\section{CONCLUSÕES}

Das análises realizadas no presente estudo verificou-se que para valores usuais de carregamento e combinações últimas pertinentes, os níveis de tensão resultantes correspondem ao regime elástico linear. Isto resulta em valores de deslocamentos nodais e momentos fletores semelhantes entre os Modelos B e C. Além disso, os momentos fletores obtidos nos Modelos B, C e D são coerentes com o Modelo $A$, que é a clássica análise elástica linear. Todavia, em alguns elementos da estrutura há divergências significativas, especialmente quando se comparam os Modelos A e D.

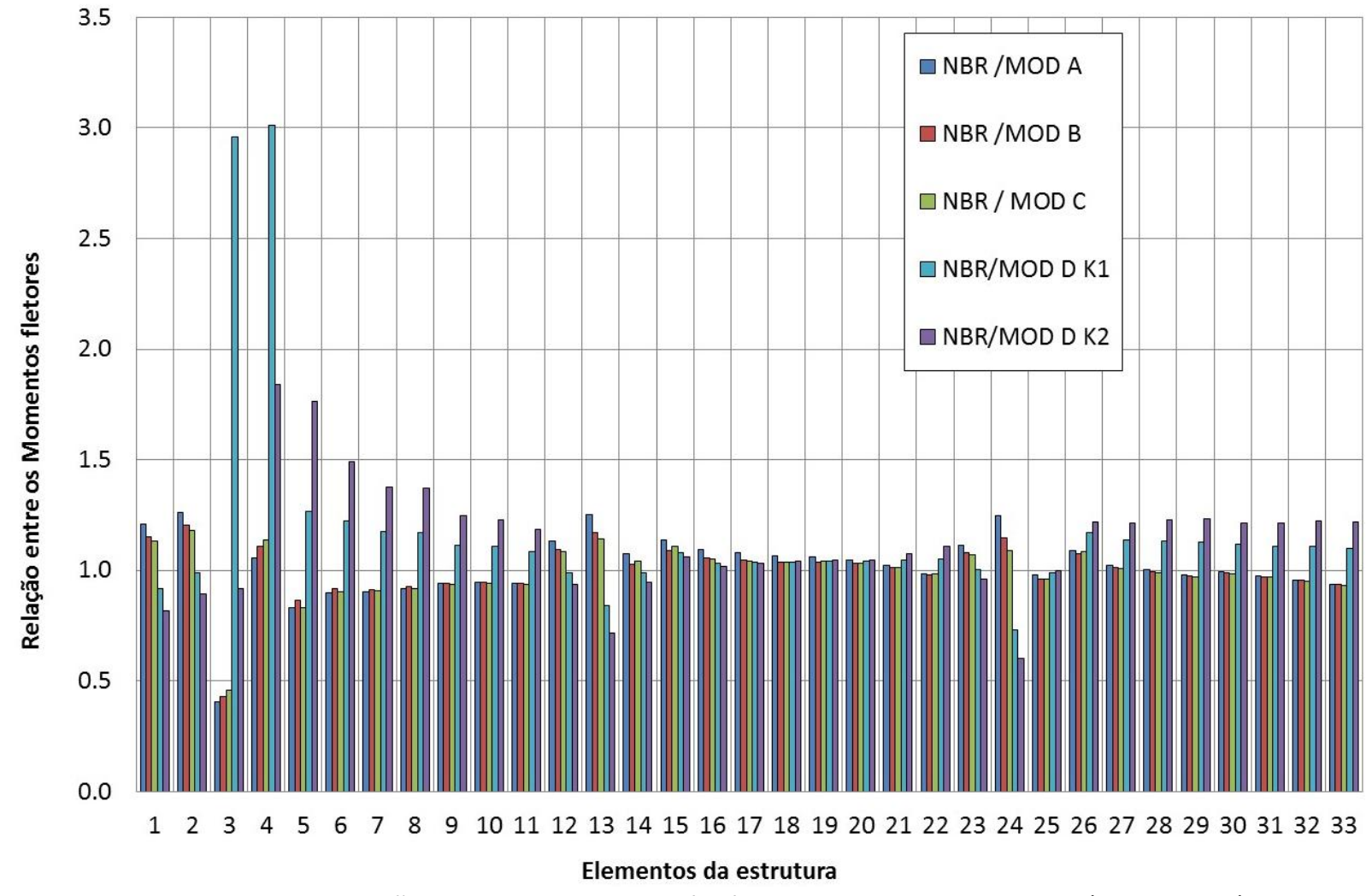

FIGURA 11: Comparação entre os Momentos obtidos via ANSYS e via NBR 8800 (ABNT, 2008). FONTE: Autoria própria. 
Quando comparados os resultados dos Modelos A, B, C e D com aqueles decorrentes da aplicação dos procedimentos de análise da NBR 8800 - (ABNT, 2008) obtém-se boa correlação. Para o exemplo 1, a mediana dos valores da relação entre os momentos segundo a NBR 8800 (ABNT, 2008) e segundo os modelos propostos foi: Modelo A - 1.11, Modelo B - 1.12, Modelo C - 1.18, Modelo D-K ${ }_{1}$ 1.32, Modelo $D-K_{2}$ - 1.33. Já para o exemplo 2 , a mediana dos valores da relação dos momentos segundo a NBR 8800 (ABNT, 2008) e segundo os modelos propostos foi: Modelo A - 1.02, Modelo B 1.01, Modelo C - 1.01, Modelo D-K 1 - 1.08, Modelo $D-K_{2}$ - 1.11. No entanto, há algumas barras onde existe grande diferença entre o modelo simplificado da NBR 8800 (ABNT, 2008) e os modelos rigorosos aqui considerados.

De uma forma geral, a proposta da NBR 8800 (ABNT, 2008) fornece bons resultados para os momentos fletores de uma estrutura com geometria e carregamentos usuais de projeto, sobretudo se houver garantia do comportamento rígido das ligações. Além disso, o presente estudo mostrou que é possível desenvolver, com modelos de barras, análises mais rigorosas considerando o comportamento não linear da estrutura e do material além da rigidez das ligações.

Por fim, é importante ter em conta que as conclusões apresentadas aqui são válidas apenas para os dois exemplos de estruturas aqui analisadas, servindo apenas de indicativo do comportamento das estruturas em geral e, portanto, não sendo possível a sua extrapolação para outras situações sem as devidas análises.

\section{REFERÊNCIAS BIBLIOGRÁFICAS}

AMERICAN INSTISTUTE OF STEEL CONSTUCTIONS. AISCLRFD. Specification for structural steel buildings. Chicago - Illinois. March, 2005.

ASSOCIAÇÃO BRASILEIRA DE NORMAS TÉCNICAS. NBR 8800: Projeto de estruturas de aço e de estruturas mistas de aço e concreto de edifícios. Rio de Janeiro, 2008.

ASSOCIAÇÃO BRASILEIRA DE NORMAS TÉCNICAS. NBR 6118: Projeto de estruturas de concreto: procedimentos. Rio de Janeiro, 2003.
Alvarenga, A. R. Aspectos Importantes na Análise Avançada com Zona Plástica de Portais Planos de Aço. Dissertação (Mestrado em Estruturas Metálicas) - Escola de Engenharia de Minas, Universidade Federal de Ouro Preto. Ouro Preto. 2005.

Alvarenga, A. R.; Silveira, R.A.M. A configuração geométrica inicial na análise avançada de portais planos de aço. Revista da Escola de Minas. v. 59, n. 2, p185-197, Jun. 2006

American Instistute of Steel Constuctions. Specification for structural steel buildings. AISC-LRFD. Chicago Illinois. 2005.

Camilo, L. H. Problema de Análise e Síntese do Método das Rotulas Plásticas. Dissertação (Mestrado) - Escola de Engenharia de São Carlos, Universidade de São Paulo. São Carlos. 1991.

Chen, W. F. Advanced analysis for structural steel building design. Frontiers of Architecture and Civil Engineering in China, v. 2, issue 3, p. 189-196, sep. 2008.

Doria, A. Análise da estabilidade de pórticos planos de aço com base no conceito de forças horizontais fictícias. Dissertação (Mestrado em Engenharia de Estruturas) Escola de Engenharia de São Carlos, Universidade de São Paulo, São Carlos. 2007.

European Committee of Standardization. ENV 1994-1-1: Eurocode 3 - Design of steel and structures, Part 1.1: General rules and rules for buildings. Bressels. 1994.

Galvão, A. S. Formulação Não Linear de Elementos Finitos para Análise. Dissertação (Mestrado). - Escola de Engenharia de Minas, Universidade Federal de Ouro Preto, Ouro Preto. 2000.

Gomes, H. C. Verificação de pilares de aço à flexão composta considerando comprimentos de flambagem, forças horizontais fictícias e análise avançada. Dissertação (Mestrado em Engenharia de Estruturas) Escola Politécnica da Universidade de São Paulo, Universidade de São Paulo, São Paulo. 2005.

Kim, S. E., Lee, D.H. Second-order distributed plasticity analysis of space steel frame. Engineering Structures ELSEVIER, v. 24, p 735-744. 2001.

King, W. S. The limit loads of steel semi-rigid frames analyzed with different methods. Computers \& Structures, v. 51, n. 5, p. 475-487.1994.

Liu, S. W.; Liu, Y. P.; Chan, S. L. Advanced analysis of hybrid steel and concrete frames: Part 2: Refined plastic hinge and advanced analysis. Journal of Constructional Steel Research, v. 70, p. 337-349, mar. 2012.

Machado, F.C.S. Análises inelástica de segunda ordem de sistemas estruturais metálicos. Dissertação (Mestrado), Escola de Engenharia de Minas , Universidade Federal de Ouro Preto, Ouro Preto. 2005. 
Pereira, M. F. ; Souza, A. S. C. Análise dos efeitos de segunda ordem em pórticos planos em aço. In: Encontro Tecnológico da Engenharia Civil e Arquitetura, 2009, Maringá. VII Encontro Tecnológico da Engenharia Civil e Arquitetura, v. 1. p. 1-10. 2009.

Reis, A.; Camotim, D. Estabilidade estrutural. 1a Edição. Lisboa: Editora Mcgraw-Hill, 2001. 470 p. 2001.

Ribeiro, L.F.L. Comportamento estrutural de ligações viga-coluna com chapa de topo: análise teórico experimental. Relatório final apresentado à FAPESP Escola de Engenharia de São Carlos, Universidade de São Paulo. 1997.

Santos, L. B. Influência da rigidez das ligações em Estruturas de Aço. Dissertação (Mestrado) - Escola de Engenharia de São Carlos, Universidade de São Paulo, São Carlos. 1998.

Silva, R. G. L. Avaliação dos efeitos de 2a ordem me edifícios de aço utilizando métodos aproximados e análise rigorosa. Dissertação (Mestrado em Engenharia de Estruturas) - Universidade Federal de Minas Gerais, Belo Horizonte.2004.

Souza, A. S. C. ; Pereira, M. F. Evolução das Técnicas de Análise e Códigos de Projeto em Análise de 2a Ordem de Estruturas Metálicas. Revista Sul-Americana de Engenharia Estrutural, v. 6, p. 1-10. 2009.

THAI, H. ; KIM, S. Large deflection inelastic analysis of space trusses using generalized displacement control method. Journal of Constructional Steel Research, v. 65, n. 10, p. 1987-1994, 2009.

Wood, B. R.; Beaulieu, D.; Adams, P. F. Column design by P-D method. Journal of the Structural Division, ASCE, v.102, n.ST2, p. 411-427, Feb 1976. 